\title{
Covering Based Pessimistic Multigranular Rough Equalities and their Properties
}

\author{
B.K.Tripathy \\ School of Computing Science and Engineering VIT University, Vellore, Tamil Nadu-632014, India \\ E-mail: tripathybk@vit.ac.in \\ S.C.Parida \\ Lecturer in Mathematics, K.B.V Mahavidyalaya, Kabisurya Nagar, Ganjam, Odisha -761104 \\ E-mail: sudamparida32@gmail.com
}

\begin{abstract}
The basic rough set theory introduced by Pawlak as a model to capture imprecision in data has been extended in many directions and covering based rough set models are among them. Again from the granular computing point of view, the basic rough sets are unigranular by nature. Two types of extensions to the context of multigranular computing are done; called the optimistic and pessimistic multigranulation by Qian et al in 2006 and 2010 respectively. Combining these two concepts of covering and multigranulation, covering based multigranular models have been introduced by Liu et al in 2012. Extending the stringent concept of mathematical equality of sets rough equalities were introduced by Novotny and Pawlak in 1985. Three more types of such approximate equalities were introduced by Tripathy in 2011. In this paper we study the approximate equalities introduced by Novotny and Pawlak from the pessimistic multigranular computing point of view and establish several of their properties. These concepts and properties are shown to be useful in approximate reasoning.
\end{abstract}

Index Terms - Rough sets, covering based rough sets, Multigranulations, Covering Based multigranulations, approximate equality.

\section{INTRODUCTION}

Data in real life are mostly imprecise in nature and so the conventional tools for formal modeling, reasoning and computing, which are crisp, deterministic and precise in characteristics, are inadequate to handle them. The notion of Rough set introduced by Pawlak [4, 5] is one of the most efficient one to handle such imprecision in data. However, its definition depends upon equivalence relations, which are relatively rare in nature. So, many extensions of basic rough sets have been proposed in the literature and covering based rough sets, which are defined by using covers instead of partitions over universes are some such extensions.

The topic of fuzzy information granulation was first proposed and discussed by L.A.Zadeh in 1979[26]. It did not attend much attention till he revived it in a seminal paper in 1997 [27]. Granulation of an object A leads to a collection of granules of A, with a granule being a clump of points (objects) drawn together by indiscernibility, similarity, proximity or functionality [25]. The theory of fuzzy information granulation (TFIG) is inspired by the ways in which humans granulate information and reason with it. Granular computing is a superset of the theory of fuzzy information granulation, rough set theory and interval computations, and is a subset of granular mathematics. An underlying idea of granular computing is the use of groups, classes or clusters of elements called granules. From a philosophical and theoretical point of view, many authors argued that information granulation is very essential to human problem solving and hence has a very significant impact on the design and implementation of intelligent systems.

From the point of view of granular computing, basic rough set theory deals with single granulation [9]. However, in some application areas we need to handle more than one granulation at a time and this necessitated the development of multi-granular rough sets (MGRS), where at least two equivalence relations are taken for granulation of a universe. This concept is further extended by considering covers and this led to the development of covering based multi granular rough sets (CBMGRS)[1, 2]. Four types of CBMGRS are defined and their properties are established.

The equality of sets used in Mathematics is too stringent a concept and also user knowledge has no role in deciding the equality of two sets. In fact, the two sets considered are identical. The real life situations are totally different from this where the user applies his/her knowledge to decide the equality of two sets.. In order to introduce human knowledge in deciding the equality of two sets and make it less stringent and more practical the notion of rough equalities were introduced by Novotny and Pawlak $[6,7,8]$ and several of their properties were established. This is an important feature as the sets considered are not equal in the crisp mathematical sense but they have close features to assume to be approximately equal. That is, basing upon our knowledge and requirement we can assume that the two sets are indistinguishable. Three more types of approximate equalities basing upon rough sets have been proposed and studied $[11,21,23]$. A comparative analysis of these 
approximate equalities is provided [11]

This paper is further organized into five sections. In the next section we provide the various definitions and notions required. In different subsections of section three we introduce basic rough equalities, covering based pessimistic multigranular rough equalities, general properties of such equalities and replacement properties of these equalities. We use several examples, which besides illustrating the computation of the lower and upper approximations of covering based multigranular rough sets are helpful in constructing counter examples to prove the properties. In the next section we provide some interpretations of the properties established in section 3 and finally provide conclusions drawn from our work. The paper ends with a bibliography of sources referred for the compilation of our work.

\section{DEFINITIONS AND NOTATIONS}

\section{A. Rough Sets}

Let $\mathrm{U}$ be a universe of discourse and $\mathrm{R}$ be an equivalence relation over $U$. By $U / R$ we denote the family of all equivalence classes of $R$, referred to as categories or concepts of $\mathrm{R}$ and the equivalence class of an element $x \in U$ is denoted by $[x]_{R}$. By a knowledge base, we understand a relational system $K=(U, P)$, where $\mathrm{U}$ is as above and $P$ is a family of equivalence relations over $\mathrm{U}$. For any subset $Q(\neq \phi) \subseteq P$, the intersection of all equivalence relations in $\mathrm{Q}$ is denoted by $\operatorname{IND}(Q)$ and is called the indiscernibility relation over Q.

Definition 1: Given any $X \subseteq U$ and $\mathrm{R} \in \mathrm{IND}$ (K), we associate two crisp sets

$$
\underline{R} X=\bigcup\{Y \in U / R: Y \subseteq X\}
$$

and

$$
\bar{R} X=\bigcup\{Y \in U / R: Y \bigcap X \neq \phi\}
$$

called the R-lower and R-upper approximations of $\mathrm{X}$ respectively.

The R-boundary of $\mathrm{X}$ is denoted by $B N_{R}(X)$ and is given by $B N_{R}(X)=\bar{R} X-\underline{R} X$. Here, $\underline{R} \mathrm{X}$ comprises of all certain elements of $\mathrm{X}$ with respect to $\mathrm{R}$ and $\bar{R} \mathrm{X}$ comprises of those elements which possibly belong to $\mathrm{X}$, with respect to $\mathrm{R}$.

We say that $X$ is rough with respect to $R$ if and only if $\underline{R} X \neq \bar{R} X$, equivalently $B N_{R}(X) \neq \phi$. $\mathrm{X}$ is said to be R-definable if and only if $\underline{R} X=\bar{R} X$, or $B N_{R}(X)=\phi$.

\section{B. Covering Based Rough Sets}

As mentioned in the introduction, Basic rough sets introduced by Pawlak have been extended in many ways. One such extension is the notion of covering based rough sets, where the notion of partitions is replaced by the general notion of covers $[1,2]$. As there are not enough of equivalence relations in real life situations the applicability of basic rough sets are limited. However, covers which can be generated by tolerance relations (only reflexive and transitive) relation and hence have better scope of application in reality. In this section we introduce the basics of these sets.

Definition 2: Let $\mathrm{U}$ be a universe and $C=\left\{C_{1}, C_{2}, \ldots C_{n}\right\}$ be a family of non-empty subsets of $U$ that are overlapping in nature. If $\mathrm{UC}=\mathrm{U}$, then $\mathrm{C}$ is called a covering of $\mathrm{U}$. The pair $(\mathrm{U}, \mathrm{C})$ is called covering approximation space.

Definition 3: For any $\mathrm{X} \subseteq \mathrm{U}$, the covering lower and upper approximations of $\mathrm{X}$ with respect to $\mathrm{C}$ can be defined as follows

$$
\begin{gathered}
\underline{C}(X)=\bigcup\left\{C_{i} \subset X, i=1,2, \ldots n\right\} \\
\bar{C}(X)=\bigcup\left\{C_{i} \cap X \neq \phi, i \in 1,2, \ldots \ldots, n\right\}
\end{gathered}
$$

The pair $(\underline{C}(X), \bar{C}(X))$ is called the covering based rough set associated with $\mathrm{X}$ with respect to cover $\mathrm{C}$ if $\underline{C}(X) \neq \bar{C}(X)$, i.e., $\mathrm{X}$ is said to with respect to $\mathrm{C}$. Otherwise $\mathrm{X}$ is said to be $\mathrm{C}$-definable.

Definition 4: Given a covering approximation space (U, C) for any $\mathrm{x} \in \mathrm{U}$, sets $m d_{c}(x)$ and $M D_{c}(x)$ are respectively called minimal and maximal descriptors of $\mathrm{x}$ with respect to $\mathrm{C}$ and are defined as

$$
\begin{aligned}
& \operatorname{md}_{C}(x)=\{M \in C / x \in M \text { and } \forall N \in C \text { suchthat }(x \in N \\
& \text { and } N \subseteq M) \text { we have } M=N\}
\end{aligned}
$$

It is the set of all minimal covering elements containing $\mathrm{x}$.

$$
\begin{aligned}
& M D_{c}(x)=\{M \in C / x \in M \text { and } \forall N \in C \text { suchthat }(x \in N \\
& \text { and } N \supseteq K \text { ) we have } M=N\}
\end{aligned}
$$

So, it is the set of all maximal covering elements containing $\mathrm{x}$.

\section{Multigranular Based Rough Sets}

In the view of granular computing, an equivalence relation on the universe can be regarded as a granulation, 
and a partition on the universe can be regarded as a granulation space $[4,5]$. There are several measures in knowledge base closely associated with granular computing, such as knowledge granulation, granulation measure, information entropy and rough entropy. Qian and Liang put forth the concepts of rough set based optimistic multigranulation and pessimistic multigranulation in 2006 [9] and 2010 [10] respectively. These notions use multiple equivalence relations simultaneously. In this paper we shall use pessimistic multigranular rough sets, which are defined as follows.

Definition 5: Let $\mathrm{K}=(\mathrm{U}, \mathrm{R})$ be a knowledge base, $\mathrm{R}$ be a family of equivalence relations, $M, N \in R$. We define the pessimistic multigranular lower approximation and upper approximation of $\mathrm{X}$ in $\mathrm{U}$ as

$$
\underline{M * N}(X)=\bigcup\left\{x /[x]_{M} \subseteq X \text { and }[x]_{N} \subseteq X\right\}
$$

and

$$
\overline{M * N}(X)=\underline{\left(M * N\left(X^{C}\right)\right)^{C}}
$$

If $\quad \underline{M * N}(X) \neq \overline{M * N}(X)$ then $\mathrm{X}$ is said to be pessimistic multigranular rough with respect $\mathrm{M}$ and $\mathrm{N}$. Else, $\mathrm{X}$ is said to be pessimistic multigranular definable with respect to $\mathrm{M}$ and $\mathrm{N}$.

\section{Covering Based Multigranular Rough Sets}

The notion of Multi-granular rough sets is extended to covering approximation space. Four types of optimistic and pessimistic covering based multigranular rough sets were defined in $[1,2,3]$ by using the notions of minimal and maximal descriptor as follows.

Let (U, C) be a covering approximation space, $C_{1}$ and $C_{2}$ be in $\mathrm{C}$ and $\mathrm{X}$ be any subset of $\mathrm{U}$. We state below the definitions of the four types of pessimistic multigranular rough sets.

Definition 6: For the first type of CBPMGRS lower and upper approximations with respect to $C_{1}$ and $C_{2}$ are defined as follows

$$
\underline{F R_{C_{1}^{*} C_{2}}}(X)=\left\{x \in U \mid \cap m d_{C_{1}}(x) \subseteq X \text { and } \cap m d_{C_{2}}(x) \subseteq X\right\} ;
$$

and

$$
\begin{gathered}
\overline{F R_{C_{1} * C_{2}}}(X)=\left\{x \in U \mid\left(\bigcap m d_{C_{1}}(x)\right) \cap X \neq \phi\right. \\
\text { or } \left.\left(\bigcap m d_{C_{2}}(x)\right) \cap X \neq \phi\right\}
\end{gathered}
$$

Definition 7: For the second type of CBPMGRS lower and upper approximations with respect to $C_{1}$ and $C_{2}$ are defined as follows

$$
\begin{gathered}
\qquad R_{C_{1} * C_{2}}(X)=\left\{x \in U \mid \cup m d_{C_{1}}(x) \subseteq X\right. \\
\text { and } \left.\bigcup m d_{C_{2}}(x) \subseteq X\right\}
\end{gathered}
$$

and

$$
\begin{gathered}
\overline{S R_{C_{1} * C_{2}}}(X)=\left\{x \in U \mid\left(\bigcup m d_{C_{1}}(x)\right) \cap X \neq \phi\right. \\
\text { or } \left.\left(\bigcup m d_{C_{2}}(x)\right) \cap X \neq \phi\right\}
\end{gathered}
$$

Definition 8: For the third type of CBMGRS lower and upper approximations with respect to $C_{1}$ and $C_{2}$ are defined as follows

$$
\begin{gathered}
\operatorname{TR}_{C_{1} * C_{2}}(X)=\left\{x \in U \mid \cap M D_{C_{1}}(x) \subseteq X\right. \\
\text { and } \left.\cap M D_{C_{2}}(x) \subseteq X\right\}
\end{gathered}
$$

and

$$
\begin{gathered}
\overline{T R_{C_{1} * C_{2}}}(X)=\left\{x \in U \mid\left(\bigcap M D_{C_{1}}(x)\right) \cap X \neq \phi\right. \\
\left.\operatorname{or}\left(\bigcap M D_{C_{2}}(x)\right) \bigcap X \neq \phi\right\}
\end{gathered}
$$

Definition 9: For the fourth type of CBMGRS lower and upper approximations with respect to $C_{1}$ and $C_{2}$ are defined as follows

$$
\begin{gathered}
\frac{L R_{C_{1}{ }^{*} C_{2}}}{}(X)=\left\{x \in U \mid \cup M D_{C_{1}}(x) \subseteq X\right. \\
\text { and } \left.\cup M D_{C_{2}}(x) \subseteq X\right\}
\end{gathered}
$$

and

$$
\begin{aligned}
\overline{L R_{C_{1} * C_{2}}}(X)= & \left\{x \in U \mid\left(\bigcup M D_{C_{1}}(x)\right) \cap X \neq \phi\right. \\
& \text { or } \left.\left(\bigcup M D_{C_{2}}(x)\right) \bigcap X \neq \phi\right\} .
\end{aligned}
$$

\section{E. Properties of Covering Based Pessimistic} Multigranular Rough Sets

Properties (15) to (20) hold for all the four types of pessimistic multigranular rough sets. However, we mention these for only the first type.

Let $(\mathrm{U}, \mathrm{C})$ be a covering approximation space and $C_{1}, C_{2} \in C$. Then for any $X, Y \subseteq U$, we have

$$
\begin{gathered}
\overline{F R_{C_{1}^{*} * C_{2}}}(\sim X)=\sim \overline{F R_{C_{1}^{*} * C_{2}}}(X) \overline{F R_{C_{1}^{*} * C_{2}}}(\sim X)=\underline{F R_{C_{1}^{*} * C_{2}}}(X) \\
X \subseteq Y \Rightarrow \underline{F R_{C_{1} * C_{2}}}(X) \subseteq \underline{F R_{C_{1} * C_{2}}}(\mathrm{Y})
\end{gathered}
$$




$$
\begin{gathered}
X \subseteq Y \Rightarrow \overline{F R_{C_{1}^{* * C_{2}}}}(X) \subseteq \overline{F R_{C_{1}^{* *} C_{2}}}(\mathrm{Y}) \\
\underline{F R_{C_{1}^{*} * C_{2}}}(X \cap Y)=\underline{F R_{C_{1}^{*} C_{2}}}(X) \cap \underline{F R_{C_{1}^{* * C_{2}}}}(Y)
\end{gathered}
$$

Proof: The proof follows from the following.

$$
\begin{aligned}
& F_{C_{1}^{*} C_{2}}(\mathrm{X} \cap \mathrm{Y})=\left\{\mathrm{x} \in \mathrm{U} \mid \cap \mathrm{md}_{C_{1}}(x) \subseteq X \cap Y \text { and } \cap \mathrm{md}_{C_{2}}(x) \subseteq X \cap Y\right\} \\
& =\left\{\mathrm{x} \in \mathrm{U} \mid \cap \mathrm{md}_{C_{1}}(x) \subseteq X \text { and } \cap \mathrm{md}_{C_{1}}(x) \subseteq Y\right. \\
& \text { and } \left.\mathrm{md}_{C_{2}}(x) \subseteq X \text { and } \cap \mathrm{md}_{C_{2}}(x) \subseteq X\right\} \\
& =\left\{\mathrm{x} \in \mathrm{U} \mid \cap \mathrm{md}_{C_{1}}(x) \subseteq X \text { and } \cap \mathrm{md}_{C_{2}}(x) \subseteq X\right. \\
& \text { and } \left.\bigcap \operatorname{md}_{C_{1}}(x) \subseteq Y \text { and } \bigcap \operatorname{md}_{C_{2}}(x) \subseteq Y\right\} \\
& =\left\{\mathrm{x} \in \mathrm{U} \mid \cap \mathrm{md}_{C_{1}}(x) \subseteq X \text { and } \cap \mathrm{md}_{C_{2}}(x) \subseteq X\right\} \\
& \text { and }\left\{\cap \operatorname{md}_{C_{1}}(x) \subseteq Y \text { and } \cap \operatorname{md}_{C_{2}}(x) \subseteq Y\right\} \\
& =\underline{F_{C_{1} * C_{2}}}(\mathrm{X}) \bigcap \underline{F_{C_{1}^{*} C_{2}}}(\mathrm{Y}) \\
& \underline{F R_{C_{1} * C_{2}}}(X \cup Y) \supseteq \underline{F R_{C_{1} * C_{2}}}(X) \cup \underline{F R_{C_{1} * C_{2}}}(Y) \\
& \overline{F R_{C_{1} * C_{2}}}(X \cup Y)=\overline{F R_{C_{1} * C_{2}}}(X) \bigcup \overline{F R_{C_{1} * C_{2}}}(Y)
\end{aligned}
$$

Proof: The proof follows from the following.

$$
\begin{aligned}
& \overline{F_{C_{1} * C_{2}}}(X \cup Y)=\left\{x \in U / \cap m d_{C_{1}}(x) \cap(X \bigcup Y) \neq \phi\right. \\
& \left.\operatorname{or} \cap m d_{C_{2}}(x) \cap(X \cup Y) \neq \phi\right\} \\
& =\left\{x \in U /\left(\bigcap m d_{C_{1}}(x) \cap X \neq \phi\right.\right. \\
& \text { or } \left.\cap m d_{C_{1}}(x) \cap Y \neq \phi\right) \text { or }\left(\bigcap m d_{C_{2}}(x) \cap X \neq \phi\right. \\
& \text { or } \left.\left.\cap m d_{C_{2}}(x) \cap Y \neq \phi\right)\right\} \\
& =\left\{x \in U /\left(\cap m d_{C_{1}}(x) \cap X \neq \phi \text { or } \bigcap m d_{C_{2}}(x) \cap X \neq \phi\right)\right. \text { or } \\
& \left.\left(\cap m_{C_{1}}(x) \cap Y \neq \phi o r \cap m d_{C_{2}}(x) \cap Y \neq \phi\right)\right\} \\
& =\overline{F_{C_{1} * C_{2}}}(X) \bigcup \overline{F_{C_{1}+C_{2}}}(Y) \\
& \overline{F R_{C_{1} * C_{2}}}(X \cap Y) \subseteq \overline{F R_{C_{1}^{*} * C_{2}}}(X) \cap \overline{F R_{C_{1}^{*} * C_{2}}}(Y)
\end{aligned}
$$

\section{APPROXIMATE EQUALITIES}

As mentioned earlier, the equality of sets or domains used in mathematics is too stringent. In most of the real life situations we often consider equality of sets or domains, as approximately equal under the existing circumstances. These existing circumstances serve as user knowledge about the set or domain. So, approximate equalities play a significant role in approximate reasoning.
Also, one can state that it mostly depends on the knowledge the assessors have about the set of domains under consideration as a whole but not on the knowledge about individuals of the set or domain.

As a step to incorporate user knowledge in considering approximate equality of sets, Novotny and Pawlak [5] introduced the following rough equalities of two sets $\mathrm{X}$ and $\mathrm{Y}$ which are subsets of $\mathrm{U}$.

Let $\mathrm{K}=(\mathrm{U}, \mathrm{R})$ be a knowledge base, $X, Y \subseteq U$ and $R \in I N D(K)$.

Definition 10: We say that,

$\mathrm{X}$ and $\mathrm{Y}$ are bottom rough equal ( $\mathrm{X} b \_R \_e q \mathrm{Y}$ ) if and only if

$$
\underline{R} X=\underline{R} Y .
$$

$\mathrm{X}$ and $\mathrm{Y}$ are top rough equal $\left(\mathrm{X} t \_R \_e q \mathrm{Y}\right)$ if and only if

$$
\bar{R} X=\bar{R} Y
$$

$\mathrm{X}$ and $\mathrm{Y}$ are rough equal (X $R \_e q \mathrm{Y}$ ) if and only if $\underline{R} X=\underline{R} Y$ and $\bar{R} X=\bar{R} Y$ i.e., $\left(\mathrm{X} b \_R \_e q \mathrm{Y}\right)$ and

$$
\left(\mathrm{X} t \_R \_e q \mathrm{Y}\right) \text {. }
$$

There are several properties of these approximate equalities established by Novotny and Pawlak [5] in the form of general and replacement properties. The replacement properties are those properties obtained from the general properties by interchanging the top and bottom rough equalities. As noted by them, all these approximate equalities of sets are relative in character; that is, sets are equal or not equal from our point of view depending on what we have about them. So, in a sense the definition of rough equality incorporates user knowledge about the universe in arriving at approximate equality of sets or domains. Some more types of such approximate equalities have been introduced by Tripathy (see for instance [11, 12, 13, 20, 21, 22]). These notions are more general and more applicable in real life situations. An example of cattle in a society is taken by him to explain the drawbacks in the earlier notion and also to establish the superiority of these new notions in the real life scenario.

In this paper we shall introduce the concepts of rough equalities in the context of covering based pessimistic multigranulations and establish their properties (both general and replacement). First type of pessimistic covering based multi granular rough set is considered and its rough equalities and equivalences are studied. The direct properties of such set are stated and proved first. Later its replacement properties are also studied and proved. To illustrate these concepts and also to provide counter examples for certain properties examples are to be provided. 
A. Covering Based Pessimistic Multigranular Approximate Equalities

We now introduce in the following different Pessimistic covering based multi granular rough equalities for first type of CBPMGRS and study their properties. For the other three types of multigranulations similar definitions hold true.

Let $C_{1}$ and $C_{2}$ be two covers on $\mathrm{U}$ and $C_{1}, C_{2} \in C$ and $X, Y \subseteq U$. Let $\mathrm{F}$ stand for first type CBPMGRS.

\section{Definition 11: We say that,}

$\mathrm{X}$ and $\mathrm{Y}$ are Covering based Pessimistic bottom rough equal to each other with respect to $C_{1}$ and $\mathrm{C}_{2}(\mathrm{X}$ $b_{-} C_{1} * C_{2} e q$ Y) if and only if

$$
\underline{F_{C_{1} * C_{2}}}(X)=\underline{F_{C_{1} * C_{2}}}(Y) .
$$

$\mathrm{X}$ and $\mathrm{Y}$ are Covering based Pessimistic top rough equal to each other with respect to $C_{1}$ and $C_{2}$ (X $t_{-} C_{1} * C_{2} e q$ Y) if and only if

$$
\overline{F_{C_{1} * C_{2}}}(X)=\overline{F_{C_{1} * C_{2}}}(Y)
$$

$\mathrm{X}$ and $\mathrm{Y}$ are Pessimistic total rough equal to each other with respect to $C_{1}$ and $C_{2}\left(\mathrm{X} r_{-} C_{1} * C_{2} \_q \mathrm{Y}\right)$ if and only if $\underline{F_{C_{1} * C_{2}}}(X)=\underline{F_{C_{1} * C_{2}}}(Y)$ and

$$
\overline{F_{C_{1} * C_{2}}}(X)=\overline{F_{C_{1} * C_{2}}}(Y) \text {. }
$$

B. Properties of Covering Based Pessimistic Multigranular Approximate Equalities

The general properties of first type of covering based rough equalities are stated, proved and substantiated few proofs with examples wherever is necessary. The properties for the other types of covering based pessimistic multigranular rough sets are the same and their proofs are similar.

Let $C_{1}$ and $C_{2}$ be two covers on $\mathrm{U}$ and $C_{1}, C_{2} \in C$ and $X, Y \subseteq U$. Let $\mathrm{F}$ denotes first type CBMGRS. Then

Property 1: $\mathrm{X} \quad b_{-} C_{1}{ }^{*} C_{2} e q \mathrm{Y}$ if and only if $X \cap Y$ $b_{-} C_{1} * C_{2} e q \mathrm{X}$ and $\mathrm{Y}$ both.

\section{Proof: (If part)}

$$
X \cap Y \quad b_{-} C_{1} * C_{2}-e q \quad X \Rightarrow \underline{F_{C_{1}{ }^{*} C_{2}}}(X \cap Y)=\underline{F_{C_{1}{ }^{*} C_{2}}}(X)
$$

and

$$
X \bigcap Y \quad b_{-} C_{1} * C_{2-} e q Y=\underline{F_{C_{1} * C_{2}}}(X \cap Y)=\underline{F_{C_{1} * C_{2}}}(Y) .
$$

So

$$
\begin{gathered}
X \cap Y b_{-} C_{1} * C_{2} e q X \text { and } Y \text { both } \Rightarrow \\
\underline{F_{C_{1} * C_{2}}}(X)=\underline{F_{C_{1} * C_{2}}}(Y), \text { that is } \mathrm{X} b_{-} C_{1} * C_{2} e q \mathrm{Y} .
\end{gathered}
$$

(Only if part) From (18) we get

$$
\underline{F_{C_{1} * C_{2}}}(\mathrm{X} \cap \mathrm{Y})=\underline{F_{C_{1}^{*} C_{2}}}(\mathrm{X}) \cap \underline{F_{C_{1} * C_{2}}}(\mathrm{Y})
$$

$$
\begin{aligned}
& \text { So, } X b_{-} C_{1} * C_{2} \text { eq } Y \Leftrightarrow \underline{F_{C_{1} * C_{2}}}(X)=\underline{F_{C_{1} * C_{2}}}(Y) \\
& \Leftrightarrow \underline{F_{C_{1}^{*} C_{2}}}(X \cap Y)=\underline{F_{C_{1}^{*} C_{2}}}(X)=\underline{F_{C_{1}^{*} C_{2}}}(Y)
\end{aligned}
$$

This completes the proof.

Property 2: $\mathrm{X} t_{-} C_{1} * C_{2} e q \mathrm{Y}$ if and only if $X \cup Y$ $t_{-} C_{1}{ }^{*} C_{2}$ eq $\mathrm{X}$ and $\mathrm{Y}$ both.

\section{Proof: (If part)}

$$
X \cup Y t_{-} C_{1} * C_{2}-e q X=>\overline{F_{C_{1} * C_{2}}}(X \cup Y)=\overline{F_{C_{1} * C_{2}}}(X)
$$

and

$$
X \bigcup Y t_{-} C_{1} * C_{2} \text { eq } Y=>\overline{F_{C_{1} * C_{2}}}(X \cup Y)=\overline{F_{C_{1} * C_{2}}}(Y)
$$

So,

$$
\begin{gathered}
X \cup Y t_{-} C_{1} * C_{2} e q \mathrm{X} \text { and } \mathrm{Y} \text { both } \Rightarrow \\
\overline{F_{C_{1} * C_{2}}}(X)=\overline{F_{C_{1} * C_{2}}}(Y)=>\mathrm{X} t_{-} C_{1} * C_{2 \_} e q \mathrm{Y} .
\end{gathered}
$$

(Only if part) From (20) we have

$$
\overline{F_{C_{1} * C_{2}}}(X \bigcup Y)=\overline{F_{C_{1} * C_{2}}}(X) \cup \overline{F_{C_{1}+C_{2}}}(Y)
$$

So, $X t_{-} C_{1} * C_{2}$ eq $Y \Leftrightarrow \overline{F_{C_{1} C_{2}}}(X \cup Y)=\overline{F_{C_{1} C_{2}}}(X)=\overline{F_{C_{1} * C_{2}}}(Y)$

This completes the proof.

Property 3: $\mathrm{X} t_{-} C_{1} * C_{2} e q X X^{\prime}$ and $\mathrm{Y} t_{-} C_{1} * C_{2} e q Y^{\prime} \Rightarrow$ $X \cup Y t_{-} C_{1} * C_{2} e q X^{\prime} \cup Y^{\prime}$.

Proof: From the proof of (Property 2) and from definition of top covering based multigranular equality we have

$$
\begin{aligned}
& \overline{F_{C_{1} * C_{2}}}(X \cup Y)=\overline{F_{C_{1} * C_{2}}}(X) \cup \overline{F_{C_{1} * C_{2}}}(Y) \\
& =\overline{F_{C_{1} * C_{2}}}\left(X^{\prime}\right) \cup \overline{F_{C_{1} * C_{2}}}\left(Y^{\prime}\right)=\overline{F_{C_{1} * C_{2}}}\left(X^{\prime} \cup Y^{\prime}\right)
\end{aligned}
$$


So,

$$
X \cup Y t_{-} C_{1} * C_{2} \_q X^{\prime} \cup Y^{\prime} .
$$

Property 4: $\mathrm{X} \quad b_{-} C_{1} * C_{2} e q X^{\prime}$ and $\mathrm{Y} b_{-} C_{1} * C_{2} e q Y^{\prime}$ $\Rightarrow X \cap Y b_{-} C_{1}{ }^{*} C_{2} e q X^{\prime} \cap Y^{\prime}$.

Proof: From the proof of (Property 1) and from definition of bottom covering based multigranular equality we have

$$
\begin{aligned}
& \underline{F_{C_{1} * C_{2}}}(X \cap Y)=\underline{F_{C_{1} * C_{2}}}(X) \cap \underline{F_{C_{1} * C_{2}}}(Y) \\
& =\underline{F_{C_{1} * C_{2}}}\left(X^{\prime}\right) \cap \underline{F_{C_{1} * C_{2}}}\left(Y^{\prime}\right)=\underline{F_{C_{1} * C_{2}}}\left(X^{\prime} \cap Y^{\prime}\right)
\end{aligned}
$$

So,

$$
X \cap Y \quad b \_C_{1} * C_{2 \_} e q X^{\prime} \cap Y^{\prime} .
$$

Property 5: $\mathrm{X} t_{-} C_{1} * C_{2} e q \mathrm{Y}=>X \cup Y^{c} t_{-} C_{1} * C_{2} \_q \mathrm{U}$

Proof: Given $\mathrm{X} t_{-} C_{1} * C_{2} e q \mathrm{Y}$

$$
\Rightarrow \overline{F_{C_{1} * C_{2}}}(X)=\overline{F_{C_{1} * C_{2}}}(Y) \text {. }
$$

Using (2.5.5) we get

$$
\begin{gathered}
\overline{F_{C_{1} * C_{2}}}\left(X \cup Y^{c}\right)=\overline{F_{C_{1} * C_{2}}}(X) \cup \overline{F_{C_{1} * C_{2}}}\left(Y^{c}\right) \\
\left.=\overline{F_{C_{1} * C_{2}}}(Y) \cup\left(\overline{F_{C_{1} * C_{2}}}(Y)\right)^{C}\right) \\
\left.=\overline{F_{C_{1} * C_{2}}}(Y) \cup\left(\overline{F_{C_{1} * C_{2}} Y \cap\left(B N_{C_{1} * C_{2}} Y\right)}\right)^{C}\right)^{C} \\
=\overline{F_{C_{1} * C_{2}}}(Y) \cup\left(\left(\overline{F_{C_{1} * C_{2}}}(Y){ }^{C} \cup\left(B N_{C_{1} * C_{2}} Y\right)^{C}\right.\right. \\
=\mathrm{U} \Rightarrow X \cup Y^{c} t_{-} C_{1} * C_{2 \_} e q \mathrm{U}
\end{gathered}
$$

Property 6: $\mathrm{X} b_{-} C_{1} * C_{2} e q \mathrm{Y} \Rightarrow X \cap Y^{c} t_{-} C_{1} * C_{2} e q \phi$

Proof: Given $\mathrm{X} b_{-} C_{1} * C_{2} e q \mathrm{Y}=>$

$$
\underline{F_{C_{1} * C_{2}}}(X)=\underline{F_{C_{1} * C_{2}}}(Y) .
$$

Now, using (18), we get

$$
\begin{gathered}
\frac{F_{C_{1} * C_{2}}}{F_{C_{1} * C_{2}}}\left(X \cap Y^{C}\right)=\underline{F_{C_{1} * C_{2}}}(X) \cap \underline{F_{C_{1} * C_{2}}}\left(Y^{C}\right)= \\
=\underline{F_{C_{C_{1} * C_{2}} * C_{2}}}(X) \cap(Y) \\
\left.=\underline{F_{C_{1} * C_{2}}}(X) \cap\left(\left(F_{C_{1} * C_{2}}(Y) \cup B N_{C_{C_{1} * C_{2}}}(Y)\right)\right)^{C} \cap\left(B N_{C_{1} * C_{2}}(Y)\right)^{C}\right)
\end{gathered}
$$

$$
\begin{gathered}
={\underline{F_{C_{1} * C_{2}}}}(X) \cap \frac{\left(F_{C_{1} * C_{2}}(X)\right)}{C} \cap\left(B N_{C_{1} * C_{2}}(Y)\right)^{C} \\
\left.=\phi \cap\left(B N_{C_{1} * C_{2}}(Y)\right)\right)^{C}=\phi \\
\quad \Rightarrow X \cap Y^{c} t_{-} C_{1} * C_{2} e q \phi
\end{gathered}
$$

Property 7: If $X \subseteq Y$ and $\mathrm{Y} t_{-} C_{1} * C_{2} e q \phi$ then $\mathrm{X} t_{-}$ $C_{1} * C_{2} e q \phi$

Proof: Given $X \subseteq Y$ and $\mathrm{Y} t_{-} C_{1}{ }^{*} C_{2 \_} e q \phi$. So we have

$$
\begin{gathered}
\overline{F_{C_{1} * C_{2}}}(Y)=\phi . \text { As } X \subseteq Y \Rightarrow X=\phi \\
\Rightarrow \overline{F_{C_{1} * C_{2}}}(X)=\phi \Rightarrow X t_{-} C_{1} * C_{2} e q \phi .
\end{gathered}
$$

Property 8: If $X \subseteq Y$ and $\mathrm{X} t_{-} C_{1} * C_{2} e q \mathrm{U}$ then $\mathrm{Y} t_{-}$ $C_{1} * C_{2} e q \mathrm{U}$

Proof: Given $X \subseteq Y$ and $\mathrm{Y} t_{-} C_{1} * C_{2} \_q$ U. So we have

$$
\overline{F_{C_{1} * C_{2}}}(X)=U
$$

Using this, as

$$
X \subseteq Y \Rightarrow \overline{F_{C_{1} * C_{2}}}(X) \subseteq \overline{F_{C_{1} * C_{2}}}(\mathrm{Y})
$$

we have $\overline{F_{C_{1} * C_{2}}}(Y)=U$. So, Y $t_{-} C_{1} * C_{2} e q \mathrm{U}$.

Property 9: $\mathrm{X} t_{-} C_{1} * C_{2} \_q \mathrm{Y}$ iff $\sim \mathrm{X} b_{-} C_{1} * C_{2} e q \sim \mathrm{Y}$

Proof: Given $\mathrm{X} t_{-} C_{1} * C_{2} e q$ Y. So, we have

$$
\overline{F_{C_{1} * C_{2}}}(X)=\overline{F_{C_{1} * C_{2}}}(Y)
$$

Hence,

$$
\begin{gathered}
\left.\left.\left.\overline{\left(F_{C_{1} * C_{2}}\right.}(X)\right)\right)^{C}=\overline{\left(F_{C_{1} * C_{2}}\right.}(Y)\right)^{C} \Rightarrow \underline{F_{C_{1} * C_{2}}}\left(X^{C}\right)=\underline{F_{C_{1} * C_{2}}}\left(Y^{C}\right) \\
\text { So, } \sim \mathrm{X} b \_C_{1} * C_{2 \_} e q \sim \mathrm{Y} .
\end{gathered}
$$

Property 10: If $\mathrm{X} b_{-} C_{1} * C_{2} e q \phi$ or $\mathrm{Y} b_{-} C_{1} * C_{2} e q \phi$ then $X \cap Y \quad b_{-} C_{1} * C_{2}$ eq $\phi$

Proof: Given $\mathrm{X} b_{-} C_{1} * C_{2} e q \phi$ or $\mathrm{Y} b_{-} C_{1} * C_{2} e q \phi$. So,

$$
F_{C_{1} * C_{2}}(X)=\phi \text { or } \underline{F_{C_{1} * C_{2}}}(Y)=\phi .
$$


Hence in any case

$$
\underline{F_{C_{1} * C_{2}}}(X) \cap \underline{F_{C_{1} * C_{2}}}(Y)=\phi .
$$

Now,

$$
\begin{gathered}
\frac{F_{C_{1} * C_{2}}}{(X \cap Y) \subseteq \underline{F_{C_{1} * C_{2}}}(X) \cap \overline{F_{C_{1} * C_{2}}}}(Y) \Rightarrow \\
\underline{F_{C_{1} * C_{2}}}(X \cap Y)=\phi=>X \cap Y \quad \overline{b_{-} C_{1} * C_{2}} e q \phi .
\end{gathered}
$$

Property 11: If $\mathrm{X} t_{-} C_{1} * C_{2} \_e q \mathrm{U}$ or $\mathrm{Y} t_{-} C_{1} * C_{2} \_e q \mathrm{U}$ then $X \cup Y t_{-} C_{1} * C_{2} e q \mathrm{U}$

Proof: Given $\mathrm{X} t_{-} C_{1} * C_{2} e q \mathrm{U}$ or $\mathrm{Y} t_{-} C_{1} * C_{2} e q \mathrm{U}$. So,

$$
\overline{F_{C_{1} * C_{2}}}(X)=\boldsymbol{U} \text { or } \overline{F_{C_{1} * C_{2}}}(Y)=U
$$

Hence, in any case

$$
\overline{F_{C_{1} * C_{2}}}(X) \cup \overline{F_{C_{1} * C_{2}}}(Y)=U
$$

Now,

$$
\begin{gathered}
\overline{F_{C_{1} * C_{2}}}(X \cup Y) \supseteq \overline{F_{C_{1} * C_{2}}}(X) \cup \overline{F_{C_{1} * C_{2}}}(Y) \\
\Rightarrow \overline{F_{C_{1} * C_{2}}}(X \cup Y)=U \\
=>X \cup Y \mathrm{t}_{-} \mathrm{C}_{1} * \mathrm{C}_{2} \text { eq } U .
\end{gathered}
$$

\section{Replacement Properties of Pessimistic Covering Based Multigranular Approximate Equalities}

These properties are also called as interchange properties. We have stated above the observation of Novotny and Pawlak in connection with holding of the properties for rough equalities when the bottom and top equalities are interchanged. They categorically told that the properties do not hold under this change. However, it is shown by Tripathy et al that some of these properties hold under the interchange where as some other hold with some additional conditions which are sufficient but not necessary. We state and prove these properties below.

Property 12: $\mathrm{X} t_{-} C_{1} * C_{2} \_e q \mathrm{Y}$ if $X \cap Y t_{-} C_{1} * C_{2} \_e q \mathrm{X}$ and $\mathrm{Y}$ both. The converse may not be true.

Proof: We have

$$
\begin{aligned}
& X \cap Y t_{-} C_{1} * C_{2-} e q X \Rightarrow \overline{F_{C_{1} * C_{2}}}(X \cap Y)=\overline{F_{C_{1} * C_{2}}}(X) \text { and } \\
& X \cap Y t_{-} C_{1} * C_{2}-e q Y \Rightarrow \overline{F_{C_{1} * C_{2}}}(X \cap Y)=\overline{F_{C_{1} * C_{2}}}(Y)
\end{aligned}
$$

So, we get

$$
\overline{F_{C_{1} * C_{2}}}(X)=\overline{F_{C_{1} * C_{2}}}(Y)
$$

\begin{tabular}{|c|c|c|c|c|c|c|c|c|}
\hline \begin{tabular}{|l} 
Eleme- \\
$\operatorname{nts}(\mathrm{x})$
\end{tabular} & $\mathrm{X}_{1}$ & $\mathrm{X}_{2}$ & $\mathrm{X}_{3}$ & $\mathrm{X}_{4}$ & $\mathrm{X}_{5}$ & $\mathrm{X}_{6}$ & $\mathrm{X}_{7}$ & $\mathrm{X}_{8}$ \\
\hline$\cap m d_{C_{1}}(x)$ & $\begin{array}{l}\left\{\mathrm{x}_{1},\right. \\
\left.\mathrm{X}_{5}\right\}\end{array}$ & $\begin{array}{c}\left\{\mathrm{X}_{2}, \mathrm{X}_{7}, \mathrm{X}\right. \\
8\}\end{array}$ & $\left\{\begin{array}{l}\left\{\mathrm{x}_{3}, \mathrm{X}_{4},\right. \\
\left.\mathrm{X}_{5}, \mathrm{X}_{6}\right\}\end{array}\right.$ & $\begin{array}{c}\left\{\mathrm{x}_{3}, \mathrm{x}_{4}, \mathrm{x}_{5}\right. \\
\left., \mathrm{x}_{6},\right\}\end{array}$ & $\left\{x_{5}\right\}$ & $\begin{array}{c}\left\{\mathrm{X}_{3}, \mathrm{X}\right. \\
4, \mathrm{X}_{5}, \mathrm{X} \\
6,\}\end{array}$ & $\begin{array}{c}\left\{\mathrm{x}_{2}, \mathrm{X}_{7},\right. \\
\left.\mathrm{x}_{8}\right\}\end{array}$ & $\begin{array}{c}\left\{\mathrm{x}_{2},\right. \\
\left.\mathrm{X}_{7}, \mathrm{X}_{8}\right\}\end{array}$ \\
\hline$\cap m d_{C_{2}}(x)$ & $\begin{array}{l}\left\{\mathrm{x}_{1},\right. \\
\left.\mathrm{x}_{6}\right\}\end{array}$ & $\begin{array}{c}\left\{\mathrm{X}_{2}, \mathrm{X}_{3}, \mathrm{X}\right. \\
4\}\end{array}$ & $\begin{array}{c}\left\{\mathrm{x}_{2}, \mathrm{X}_{3},\right. \\
\left.\mathrm{X}_{4}\right\}\end{array}$ & $\begin{array}{c}\left\{\mathrm{x}_{2}, \mathrm{X}_{3}, \mathrm{X}_{4}\right. \\
\}\end{array}$ & $\begin{array}{l}\left\{\mathrm{x}_{5},\right. \\
\left.\mathrm{X}_{6}\right\}\end{array}$ & $\left\{\mathrm{x}_{6}\right\}$ & $\begin{array}{l}\left\{\mathrm{x}_{7},\right. \\
\left.\mathrm{x}_{8}\right\}\end{array}$ & $\begin{array}{l}\left\{\mathrm{x}_{7},\right. \\
\left.\mathrm{X}_{8}\right\}\end{array}$ \\
\hline
\end{tabular}

which implies that $\mathrm{X} t \_C_{1} * C_{2} \_e q \mathrm{Y}$.
To show that the converse may not be true, we provide one example.

Let

$$
\mathrm{U}=\left\{x_{1}, x_{2}, x_{3}, x_{4}, x_{5}, x_{6}, x_{7}, x_{8}\right\}
$$

We define two covers $C_{1}$ and $C_{2}$ as follows.

$$
\begin{aligned}
& U / C_{1}=\left\{\left\{x_{1}, x_{5}\right\},\left\{x_{3}, x_{4}, x_{5}, x_{6}\right\},\left\{x_{2}, x_{7}, x_{8}\right\}\right\} \text { and } \\
& U / C_{2}=\left\{\left\{x_{1}, x_{6}\right\},\left\{x_{2}, x_{3}, x_{4}\right\},\left\{x_{5}, x_{6}\right\},\left\{x_{7}, x_{8}\right\}\right\}
\end{aligned}
$$

The minimum descriptions for the different elements are tabulated below.

Table 1. The minimal descriptions

Let us take $\mathrm{X}=\left\{x_{1}, x_{4}\right\}$ and $\mathrm{Y}=\left\{x_{1}, \mathrm{x}_{6}\right\}$ Then

$$
\overline{F_{C_{1} * C_{2}}}(X)=\left\{x_{1}, x_{3}, x_{4}, x_{6}\right\}=\overline{F_{C_{1} * C_{2}}}(\mathrm{Y}) .
$$

So, $\mathrm{X} t_{-} C_{1} * C_{2} \_e q$ Y. But, $X \bigcap Y=\left\{x_{1}\right\}$. So,

$$
\overline{F_{C_{1} * C_{2}}}(X \cap Y)=\left\{x_{1}\right\} \neq \overline{F_{C_{1} * C_{2}}}(\mathrm{X}) \text { or } \overline{F_{C_{1} * C_{2}}}(\mathrm{Y}) .
$$

Hence, $X \cap Y$ is not $t_{-} C_{1} * C_{2} \_q$ to $\mathrm{X}$ and $\mathrm{Y}$ both.

Property 13: $\mathrm{X} b_{-} C_{1} * C_{2} \_e q \mathrm{Y}$ if $X \cup Y b_{-} C_{1} * C_{2} \_e q \mathrm{X}$ and $\mathrm{Y}$ both. Converse need not be true

Proof: We have

$$
\begin{aligned}
& X \cup Y b_{-} C_{1} * C_{2}-e q X \Rightarrow \underline{F_{C_{1} * C_{2}}}(X \cup Y)=\underline{F_{C_{1} * C_{2}}}(X) \\
& \text { and } X \cup Y b_{-} C_{1} * C_{2}-e q Y \Rightarrow \underline{F_{C_{1} * C_{2}}}(X \cup Y)=\underline{F_{C_{1} * C_{2}}}(\mathrm{Y})
\end{aligned}
$$

So, we get $F_{C_{1} * C_{2}}(X)=F_{C_{1} * C_{2}}(Y)$, which implies that $\mathrm{X}$ b_ $C_{1} * C_{2} e q \mathrm{Y}$.

The converse may not be true. It can be seen from the following example. We continue with the example in (3.4.1). Here we take

$$
\mathrm{X}=\left\{x_{1}, x_{4}, x_{5}, x_{6}\right\} \text { and } \mathrm{Y}=\left\{x_{1}, x_{3}, x_{5}\right\}
$$

Then 


$$
\underline{F_{C_{1} * C_{2}}}(X)=\left\{x_{1}, x_{5}\right\}=\underline{F_{C_{1} * C_{2}}}(Y) \text {. }
$$

But

$$
\mathrm{X} \cup \mathrm{Y}=\left\{x_{1}, x_{3}, x_{4}, x_{5}, x_{6}\right\} . \text { So, }
$$

$\underline{F_{C_{1} * C_{2}}}(X \cup Y)=\left\{x_{1}, x_{3}, x_{4}, x_{5}, x_{6}\right\} \neq \underline{F_{C_{1} * C_{2}}}(\mathrm{X})$ or $\underline{F_{C_{1} * C_{2}}}(\mathrm{Y})$

Hence, $X \bigcup Y$ not $b_{-} C_{1} * C_{2} e q \mathrm{X}$ or $\mathrm{Y}$.

Property 14: $\mathrm{X} \mathrm{b}_{-} C_{1} * C_{2} \_e q X^{\prime}$ and $\mathrm{Y} \mathrm{b}_{-} C_{1} * C_{2} \_e q Y^{\prime}$ may not imply that $X \bigcup Y \mathrm{~b}_{-} C_{1} * C_{2} e q X^{\prime} \cup Y^{\prime}$.

Proof: in order to prove this we consider the example provided in (property 12).

Let us take $\mathrm{X}=\left\{x_{1}, x_{5}, x_{6}\right\}, \mathrm{X}^{\prime}=\left\{x_{1}, \mathrm{X}_{4}, x_{5}, x_{6}\right\}, \mathrm{Y}$ $=\left\{x_{1}, \mathrm{x}_{4}, x_{5}, x_{6}\right\}$ and $\mathrm{Y}^{\prime}=\left\{x_{1}, \mathrm{x}_{2}, \mathrm{x}_{3}, x_{5}, x_{6}\right\}$. Then

$$
\underline{F_{C_{1} * C_{2}}}(X)=\underline{F_{C_{1} * C_{2}}}\left(X^{\prime}\right)=\underline{F_{C_{1} * C_{2}}}(\mathrm{Y})=\underline{F_{C_{1} * C_{2}}}\left(\mathrm{Y}^{\prime}\right)=\left\{x_{1}, x_{5}\right\}
$$

But,

$X \bigcup Y=\left\{x_{1}, x_{4}, x_{5}, x_{6}\right\}$ and $X^{\prime} \cup Y^{\prime}=\left\{x_{1}, x_{2}, x_{3}, x_{4}, x_{5}, x_{6}\right\}$ So,

and

$$
\underline{F_{C_{1} * C_{2}}}(X \cup Y)=\left\{x_{1}, x_{5}\right\}
$$

$$
\underline{F_{C_{1} * C_{2}}}\left(X^{\prime} \cup Y^{\prime}\right)=\left\{x_{1}, x_{3}, x_{4}, x_{5}, x_{6}\right\}
$$

It is clear from the example that the property holds.

Property 15: $\mathrm{X} t_{-} C_{1} * C_{2} \_e q X^{\prime}$ and $\mathrm{Y} t_{-} C_{1} * C_{2} \_e q Y^{\prime}$ may not imply that $X \cap Y t_{-} C_{1} * C_{2} e q X^{\prime} \cap Y^{\prime}$

Proof: We construct an example to show this negative result from the example given in (property 12).

$$
\text { Let } X=\left\{x_{1}, x_{4}\right\} X^{\prime}=\left\{x_{1}, x_{6}\right\}, Y=\left\{x_{3}, x_{6}, x_{7}\right\} \text { and } Y^{\prime}=\left\{x_{6}, x_{8}\right\} \text {. }
$$

Then

$$
\overline{F_{C_{1} * C_{2}}}(X)=\left\{x_{1}, x_{3}, x_{4}, x_{6}\right\}=\overline{F_{C_{1} * C_{2}}}\left(X^{\prime}\right)
$$

and

$$
\overline{F_{C_{1} * C_{2}}}(Y)=\left\{x_{2}, x_{3}, x_{4}, x_{6}, x_{7}, x_{8}\right\}=\overline{F_{C_{1} * C_{2}}}\left(Y^{\prime}\right) .
$$

However,

$$
\overline{F_{C_{1} * C_{2}}}\left(X \cap X^{\prime}\right)=\phi
$$

and

$$
\overline{F_{C_{1} * C_{2}}}\left(Y \cap Y^{\prime}\right)=\left\{x_{3}, x_{4}, x_{6}\right\}
$$

So, our claim is true.

Property 16: $\mathrm{X} b_{-} C_{1} * C_{2} e q \mathrm{Y}=>X \cup Y^{C} b_{-} C_{1} * C_{2} \_q$ U

Proof: $\mathrm{X} b \_C_{1} * C_{2} \_e q \mathrm{Y}=>\underline{F_{C_{1} * C_{2}}}(X)=\underline{F_{C_{1} * C_{2}}}(Y)$.

But from (19) we have

$$
\underline{F_{C_{1} * C_{2}}}(X \cup Y) \supseteq \underline{F_{C_{1} * C_{2}}}(X) \underline{\bigcup F_{C_{1} * C_{2}}}(Y) .
$$

Thus we have from this the following

$$
\begin{aligned}
& \underline{F_{C_{1} * C_{2}}}\left(X \cup Y^{C}\right) \supseteq \underline{F_{C_{1} * C_{2}}}(X) \cup \underline{F_{C_{1} * C_{2}}}\left(Y^{C}\right) \\
& =\underline{F_{C_{1} * C_{2}}}(Y) \cup\left(\overline{F_{C_{1} * C_{2}}}(Y)\right){ }^{C} \\
& \left.=\underline{F_{C_{1} * C_{2}}}(Y) \cup\left(\underline{\left(F_{C_{1} * C_{2}}\right.}(Y) \cup B N_{C_{1} * C_{2}} Y\right)\right)^{C} \\
& \left.=\underline{F_{C_{1} * C_{2}}}(Y) \cup\left({\underline{\left(F_{C_{1} * C_{2}}\right.}}(Y)\right){ }^{C} \cap\left(B N_{C_{1} * C_{2}} Y\right)^{C}\right) \\
& \subseteq \underline{F_{C_{1}+C_{2}}}(Y) \bigcup\left(U-\underline{F_{C_{1}+C_{2}}}(Y)\right)=U \Rightarrow X \bigcup Y^{C} b_{-} C_{1}+C_{2} e q \mathrm{U}
\end{aligned}
$$

Property 17: $\mathrm{X} t_{-} C_{1} * C_{2} e q \mathrm{Y} \Rightarrow X \cap Y^{C} b_{-} C_{1} * C_{2} e q$ $\phi$

Proof: $\mathrm{X} t_{-} C_{1} * C_{2} e q \mathrm{Y} \Rightarrow \overline{F_{C_{1}+C_{2}}}(X)=\overline{F_{C_{1}+C_{2}}}(Y)$. Now, from (2.5.4) we get

$$
\begin{gathered}
\quad \underline{F_{C_{1} * C_{2}}}\left(X \cap Y^{C}\right)=\underline{F_{C_{1} * C_{2}}}(X) \cap \underline{F_{C_{1} * C_{2}}}\left(Y^{C}\right) \\
=\underline{F_{C_{1} * C_{2}}}(X) \cap\left(\overline{F_{C_{1} * C_{2}}}(Y)\right){ }^{C}=\underline{F_{C_{1} * C_{2}}}(X) \cap\left(\overline{F_{C_{1} * C_{2}}}(\mathrm{X})\right){ }^{C}= \\
\underline{F_{C_{1} * C_{2}}}(X) \cap\left(\left(\underline{F_{C_{1} * C_{2}}}(X) \cup B N \overline{C_{1} * C_{2}}(\mathrm{X})\right)^{C}=\right. \\
\left.\underline{F_{C_{1} * C_{2}}}(X) \cap\left(\left(\underline{F_{C_{1} * C_{2}}}(X)\right)^{C}\right)=\phi \cap \underline{\left(F_{C_{1} * C_{2}}\right.}(X)\right)^{C}=\phi \\
\Rightarrow X \cap Y^{C} \mathrm{~b}_{-} C_{1} * C_{2} e q \phi .
\end{gathered}
$$

Property 18: If $X \subseteq Y$ and $\mathrm{Y} b_{-} C_{1} * C_{2} e q \phi$ then $\mathrm{X} b_{-}$ $C_{1} * C_{2} \_q \phi$

Proof: $\mathrm{Y} b_{-} C_{1} * C_{2} \_e q \phi \underline{F_{C_{1}+C_{2}}}(Y)=\phi$. Also

$$
X \subseteq Y \Rightarrow>\underline{F_{C_{1}+C_{2}}}(X) \subseteq \underline{F_{C_{1}+C_{2}}}(\mathrm{Y})=\phi
$$


So, $\mathrm{X} b_{-} C_{1} * C_{2} e q \phi$

Property 19: If $X \subseteq Y$ and $\mathrm{X} b_{-} C_{1} * C_{2} e q \mathrm{U}$ then $\mathrm{Y} b_{-}$ $C_{I} * C_{2} e q \mathrm{U}$

Proof: $\mathrm{Y} b_{-} C_{1} * C_{2 \_} e q \mathrm{U} \Rightarrow \underline{F_{C_{1}+C_{2}}}(X)=U$. Also,

$$
\begin{aligned}
& X \subseteq Y \Rightarrow \underline{F_{C_{1}+C_{2}}}(X) \subseteq \underline{F_{C_{1}+C_{2}}}(Y) \text {. So, } \underline{F_{C_{1}+C_{2}}}(Y)=U \text {. } \\
& \Rightarrow \mathrm{Y} b_{-} C_{1}+C_{2} e q \mathrm{U} \text {. }
\end{aligned}
$$

Property 20: $\mathrm{X} \quad b_{-} C_{1} * C_{2} e q \mathrm{Y}$ iff $X^{C} t_{-} C_{1} * C_{2} e q$ $Y^{C}$

Proof: $\mathrm{X} b_{-} C_{1} * C_{2} e q \mathrm{Y} \Leftrightarrow \underline{F_{C_{1}+C_{2}}}(X)=\underline{F_{C_{1}+C_{2}}}(Y)$

$$
\begin{aligned}
& \left.\Leftrightarrow \overline{F_{C_{1} * C_{2}}}\left(X^{C}\right)\right)^{C}=\left(\overline{F_{C_{1} * C_{2}}}\left(Y^{C}\right)\right)^{C} \Leftrightarrow \overline{F_{C_{1} * C_{2}}}\left(X^{C}\right)= \\
& \left.\overline{F_{C_{1} * C_{2}}(Y}{ }^{C}\right) \Leftrightarrow X^{C} t_{-} C_{1} * C_{2} \text { eq } Y^{C}
\end{aligned}
$$

In a similar way converse will also be proved.

Property 21: If $\mathrm{X} t_{-} C_{1}{ }^{*} C_{2} e q \phi$ or $\mathrm{Y} t_{-} C_{1} * C_{2} e q \phi$ then $X \cap Y t_{-} C_{1} * C_{2} e q \phi$

\section{Proof:}

$$
\begin{aligned}
& X t_{-} C_{1} * C_{2-} \text { eq } \% \text { or } Y t_{-} C_{1} * C_{2-} e q \phi \\
& \Rightarrow \overline{F_{C_{1}+C_{2}}}(X)=\phi \text { or } \overline{F_{C_{1}+C_{2}}}(Y)=\phi .
\end{aligned}
$$

In any case, we have

$$
\overline{F_{C_{1} * C_{2}}}(X) \cap \overline{F_{C_{1} * C_{2}}}(Y)=\phi
$$

But then using (21)

$$
\overline{F_{C_{1} * C_{2}}}(X \cap Y) \subseteq \overline{F_{C_{1} * C_{2}}}(X) \cap \overline{F_{C_{1} * C_{2}}}(Y)
$$

So,

$$
\overline{F_{C_{1} * C_{2}}}(X \cap Y)=\phi \text {. Hence, } X \cap Y \quad t \_C_{1}+C_{2 \_} e q \phi
$$

Property 22: If $\mathrm{X} b_{-} C_{1} * C_{2-} e q \mathrm{U}$ or $\mathrm{Y} b_{-} C_{1} * C_{2} e q \mathrm{U}$ then $X \cup Y \quad b_{-} C_{1} * C_{2} \_q \mathrm{U}$

Proof: $\mathrm{X} b_{-} C_{1} * C_{2} e q \mathrm{U}$ or $\mathrm{Y} b_{-} C_{1} * C_{2} \_q \mathrm{U}=>$

$$
\underline{F_{C_{1}+C_{2}}}(X)=U \text { or } \underline{F_{C_{1}+C_{2}}}(Y)=U \text {. }
$$

So, in any case we have

$$
\underline{F_{C_{1}+C_{2}}}(X) \cup \underline{F_{C_{1}+C_{2}}}(Y)=U .
$$

But then using (19)

$$
\underline{F_{C_{1}+C_{2}}}(X \cup Y) \supseteq \underline{F_{C_{1}+C_{2}}}(X) \underline{\cup F_{C_{1}+C_{2}}}(Y)=>\underline{F_{C_{1}+C_{2}}}(X \cup Y)=U .
$$

So,

$$
X \bigcup Y \quad b_{-} C_{1} * C_{2} e q \mathrm{U} \text {. }
$$

The following example shows that converse need not be true.

$$
\begin{aligned}
& \text { Let } \mathrm{X}=\left\{x_{3}, x_{6}\right\}, Y=\left\{x_{3}, x_{7}\right\} \text { and } X \cup Y=\left\{x_{3}, x_{6}, x_{7}\right\} \\
& \underline{F_{C_{1}+C_{2}}}(X)=\left\{x_{3}\right\} \text { and } \underline{F_{C_{1}+C_{2}}}(Y)=\left\{x_{3}\right\} \quad \underline{F_{C_{1}+C_{2}}}(X \cup Y)=\left\{x_{3}, x_{6}, x_{7}\right\} \\
& \text { Thus } \underline{F_{C_{1}+C_{2}}}(X \cup Y) \neq \underline{F_{C_{1}+C_{2}}}(X) \text { and } \underline{F_{C_{1}+C_{2}}}(X \cup Y) \neq \underline{F_{C_{1}+C_{2}}}(Y) \\
& \text { though } \underline{F_{C_{1}+C_{2}}}(X)=F_{C_{1}+C_{2}}(Y)
\end{aligned}
$$

\section{SOME INTERPRETATIONS}

The covering based pessimistic multigranular lower approximation comprises of those elements which have the intersection of their minimal descriptors with respect to both the granulations contained in the set. The minimal descriptors comprises of the intersection of all the smallest covers containing the element. So, in a sense the lower approximation contains those elements which belong to the core of the granulations with respect to both the granular structures. Similarly, the covering based pessimistic multigranular upper approximations comprises of those elements which have nonempty intersection with the intersection of the minimal descriptors with respect to both the granulations. So, the upper approximation consists of elements which have some commonality with the core of the granulations with respect to both the granular structures. These two concepts provide the bounds of knowledge associated with the two granular structures on a set. Thus the rough equalities also provide the equalities with respect to the two concepts for two sets and are more general.

\section{CONCLUSIONS}

The notions of approximate equalities first introduced by Novotny and Pawlak have attracted very little attention so far, in spite of its importance in using user knowledge in deciding equality of concepts. But this is done in day to day real life situations more often than not. These notions have been extended by Tripathy in several 
of his papers. Extensions of these results to the multigranulation scenario have also been done. Covering based multigranulations are recent additions to multigranulations. Extensions of the concepts of approximate equalities were on the card. In this paper we introduced the concepts of covering based pessimistic multigranular rough equalities and established several of their direct and replacement properties. We provided suitable examples for illustrations and complete proofs as counter examples. These types of approximate equalities can be applied in approximate reasoning in more general context than the classifications.

\section{REFERENCES}

[1] Lin GP, Qian YH, Li J.J.: a covering-based pessimistic multi-granulation rough set, in: Proceedings of International Conference on Intelligent Computing, August 11-14, (2011), Zhengzhon, China.

[2] Liu, C. H. and Miao, D.Q.: Covering rough set model based on multi-granulations, in: Proceedings of Thirteenth International Conference on Rough Sets, Fuzzy Set, Data Mining and Granular Computing, LNCS (LNAI) 6743, (2011), pp. 87-90.

[3] Liu C. L., Miao D. and Quain J.: On multi-granulation covering rough sets, International Journal of Approximate Reasoning, November (2012).

[4] Pawlak, Z.: Rough sets, Int. jour. of Computer and Information Sciences, 11, (1982), pp.341-356.

[5] Pawlak, Z.: Rough sets: Theoretical aspects of reasoning about data, Kluwer academic publishers (London), (1991).

[6] Novotny, M. and Pawlak, Z.: Black Box Analysis and Rough Top Equality, Bull. Polish Acad. Sci. Math., 33, (1985), pp.105-113.

[7] Novotny, M. and Pawlak, Z.: Rough Top Equalities and Rough Bottom Equalities, Bull. Polish Acad. Sci. Math., 33, (1985), pp.91-97.

[8] Novotny, M. and Pawlak, Z.: On Rough Equalities, Bull. Polish Acad. Sci. Math., 33, (1985), pp.99-104.

[9] Qian, Y. H and Liang, J.Y.: Rough set method based on Multi-granulations, Proceedings of the 5th IEEE Conference on Cognitive Informatics, (2006), 1, pp.297 304.

[10] Qian, Y. H., Liang, J. Y and Dang, C.Y.: Pessimistic rough decision, proceedings of RST 2010, Zhoushan, China, (2010), pp.440-449.

[11] Tripathy, B. K.: An Analysis of Approximate Equalities based on Rough Set Theory, International journal of Advances in Science and Technology,vol.31, June, (2011),pp.23-36.

[12] Tripathy, B.K., Rawat, R., Divya Vani Y, and Parida, S. C.: Approximate Rough Equalities, International Journal of Intelligent Systems and Applications 6: (2014), pp.6976.

[13] Tripathy, B.K. and Mitra, A.: On Approximate Equivalences of Multigranular Rough Sets and Approximate Reasoning, International Journal of Information Technology and Computer Science, 10, (2013), pp.103-113.
[14] Tripathy, B. K. and Panda, G.K.: Approximate Equalities on Rough Intuitionistic Fuzzy Sets and an Analysis of Approximate Equalities, International Journal of Computer Science Issues (IJCSI) ,9, (2012), pp.371-380.

[15] Tripathy, B. K. and Nagaraju, M.: On Some Topological Properties of Pessimistic Multigranular Rough Sets, International Journal of Intelligent Systems and Applications, 8, (2012), pp.10-17.

[16] Tripathy, B. K. and Nagaraju, M.: A Comparative Analysis of Multigranular approaches and on Topological Properties of Incomplete Pessimistic Multigranular Rough Fuzzy sets, International Journal of Intelligent Systems and Applications, 11, (2012), pp.99-109.

[17] Tripathy, B. K. and Raghavan, R.: On Some Topological Properties of Multigranular Rough Sets, Journal of Advances in Applied science Research, Vol.2, no.3: (2011), pp.536-543.

[18] Tripathy, B K and Mitra A.: Topological Properties of Rough Sets and their Applications, International Journal of Granular Computing, Rough Sets and Intelligent Systems (IJGCRSIS), (Switzerland),vol.1, no..4 (2010), pp.355-369.

[19] Tripathy, B. K.: Rough sets on Fuzzy approximation spaces and Intuitionistic Fuzzy approximation spaces, Springer International studies in computational intelligence, vol.174, Rough Set Theory: A True landmark in Data Analysis, Ed: A. Abraham, R.Falcon and R.Bello, (2009), pp.3 - 44.

[20] Tripathy, B. K. and Panda, G.K.: On Covering Based Approximations of Classifications of Sets", IEA/AIE 2009, LNAI 5579, (2009), pp.777-786.

[21] Tripathy, B. K.: On Approximation of Classifications, Rough Equalities and Rough Equivalences", Springer International studies in computational intelligence, vol.174, Rough Set Theory: A True landmark in Data Analysis, Ed: A. Abraham, R.Falcon and R.Bello: (2009), pp. $85-133$

[22] Tripathy, B. K. and Tripathy, H.. K.: Covering Based Rough Equivalence of Sets and Comparison of Knowledge", Proceedings of the IACSIT Spring Conference 2009, Singapore, 17-20 April 2009, pp.303307.

[23] Tripathy, B. K., Mitra, A. and Ojha, J.: On Rough Equalities and Rough Equivalence of Sets", ", RSCTC 2008-Akron, U.S.A., Springer-Verlag Berlin Heidelberg 2008, LNAI 5306: pp.92-102.

[24] Yao, Y.Y and Yao, B.: Covering based rough set approximations, Information Sciences, 200, (2012), pp.91-107.

[25] Yao, Y. Y.: Perspectives of Granular Computing", Proceedings of 2005 IEEE International Conference on Granular Computing, I: (2005), pp. 85-90.

[26] Zadeh, L.A: Fuzzy sets and information granularity, In: Advances in Fuzzy set Theory and Applications", N.Gupta, R.Regade and R.Yager (Eds.), (1979), pp.3-18.

[27] Zadeh, L.A: Towards a theory of fuzzy information granulation and its centrality in human reasoning and fuzzy logic, Fuzzy sets and systems, 19, (1997),pp.111127

[28] Zakowski W.: Approximations in the space (U II), Demonstration Mathematics, 16, (1998), pp.761-769. 


\section{Authors' Profiles}

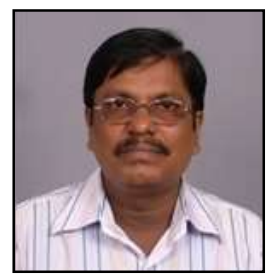

B.K.Tripathy is a senior professor in the school of computing sciences and engineering, VIT University, Vellore since 2007. He has produced $20 \mathrm{PhDs}$, 13 M.Phils and 02 M.S students so far. He has published more than 250 papers in different international journals, conference proceedings and edited research volumes. He has edited two research volumes for the IGI publications and coauthored a book on Soft Computing. He is in the editorial board or review panel of over 60 journals including Springer, Science Direct, IEEE and World Scientific publications. He is a life member/ senior member/member of 21 international forums including ACM, IEEE, ACEEE and CSI. His current interest includes Fuzzy sets and systems, Rough sets and knowledge engineering, Multiset theory, List theory, Data clustering and database anonymisation, content based learning, Neighbourhood systems, Soft set analysis, Image processing ,cloud computing and Social Network Analysis.

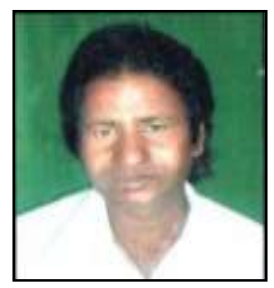

S.Ch.Parida is a lecturer in Mathematics in KBV College, Odisha. He is pursuing his $\mathrm{Ph} . \mathrm{D}$ under the supervision of Dr.B.K.Tripathy. He has a few papers published in international journals. His current research interest includes Rough sets and knowledge engineering and soft computing.

How to cite this paper: B.K.Tripathy, S.C.Parida,"Covering Based Pessimistic Multigranular Rough Equalities and their Properties", International Journal of Information Technology and Computer Science(IJITCS), Vol.8, No.4, pp.52-62, 2016. DOI: $10.5815 /$ ijitcs.2016.04.07 